\title{
Association Between Schiffs and Mannich Bases of 5-Substituted-1h Indole-2, 3 Diones and Evaluation of Antiepileptic Activity: Findings from PTZ Model Casual Pathway-Based Multi-Site Recruitment
}

\author{
Rahul Hajare* \\ Indian Council of Medical Research, India
}

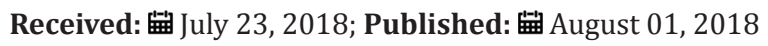

*Corresponding author: Rahul Hajare, Department of Quality Assurance, Pune University Pune, India

\begin{abstract}
We report here novel Schiff bases of 5-substituted-1H Indole-2, 3 Dione comparing modified with aromatic primary amine and hydrazine. A recombinant with Mannich base has been synthesized. A structure of the synthesized compounds was analyzed for structure confirmed by mass spectral data. Compound 1a-p has screened for anti-convulsant activity, increased in latency time and reduction in duration of convulsion and standard drug when compared, sodium valporate $300 \mathrm{mg} / \mathrm{kg}$. PTZ dose of $90 \mathrm{mg} / \mathrm{kg}$ produced severe clonic convulsions in $88 \%$ of the mice injected, with a mean onset time of $3.61 \pm 0.5$ min and $80 \%$ mortality. Clonic convulsive activity lasted $2.3 \pm 0.45 \mathrm{~min}$ and has postictal period of decreased motor activity and subsequently. Test result revealed that compounds at dose of $300 \mathrm{mg} / \mathrm{kg}$, the severity and duration of the convulsive episode following PTZ has not observed.
\end{abstract}

Keywords: 1H Indole-2, 3 Diones; Schiffs base; Mannich base; Anti-convulsant

\section{Introduction}

Epilepsy has accepted group of disorders in which there are recurrent episodes of altered cerebral function associated with paroxysmal excessive \& hypersynchronus discharge of cerebral neurons [1]. The clinical accompaniments of those episodes seizures vary in manifestation from brief lapses of awareness to prolonged bouts of unconsciousness incontinence. It has episode of neurological dysfunction a seizure. Isatin (1H-indole-2, 3-dione) and scaffold demonstrated a diverse array of biological activities. It has 5-halo derivatives have reacted to form the Schiff's base, Mannich bases to form $\mathrm{C}-\mathrm{N}, \mathrm{C}=\mathrm{N}$ bonds, from the spectral studies, Isatin undergo reaction at $\mathrm{C}-3$ and $\mathrm{N}-1$ position and showed the anti-convulsant activity.

\section{R \& D of Iso nitroso acetanilide (Scheme I)}

In round bottom flask took $9 \mathrm{~g}(0.05$ moles $)$ of chloral hydrate in $86 \mathrm{~mL}$ of water added mixture $7 \mathrm{~g}$ of sodium sulfate and solution of $3.1 \mathrm{~g}$ ( $0.03 \mathrm{moles})$ of aniline (4-halo substituted derivatives) in $30 \mathrm{~mL}$ of water containing 4-5 $\mathrm{mL}$ of $\mathrm{HCl}$ added (dissolved amines).
Finally, the solution of hydroxylamine in $50 \mathrm{~mL}$ of water added in the flask. Heat the mixture at $45^{\circ} \mathrm{C}$ to dissolved precipitate and then boiled the solution vigorously for 1-2 min. Cooled the solution to room temperature. The precipitate obtained has filtered off. The yield has $80 \%$ (Scheme 1).

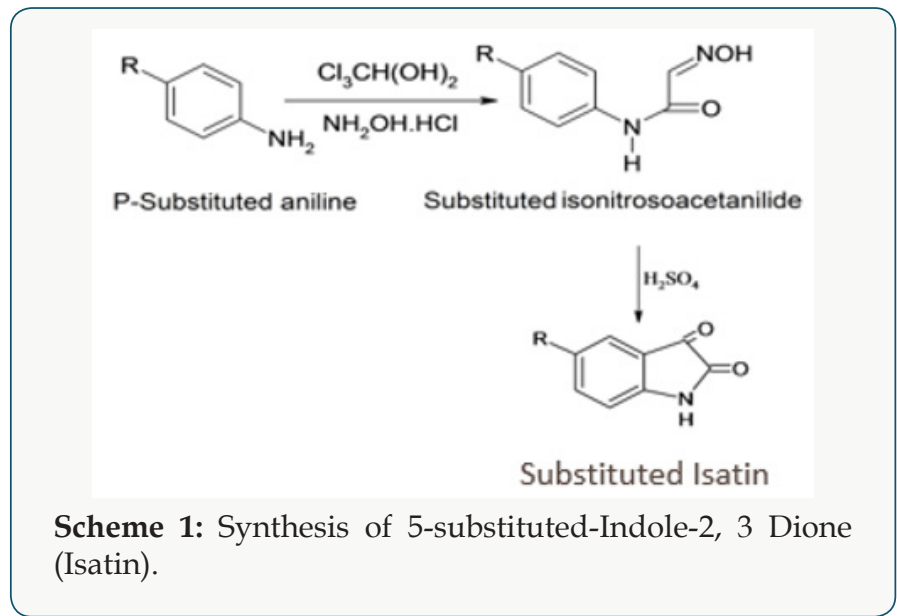




\section{R \& D of 5-Substituted-Indole-2, 3 Dione (Scheme I)}

Heat the $3 \mathrm{~mL}$ of concentrated Sulphuric-acid at $70{ }^{\circ} \mathrm{C}$. To this added $1 \mathrm{~g}$ of iso nitroso acetanilide with vigorous stirring. Heat the mixture to $80{ }^{\circ} \mathrm{C}$ for $1-2 \mathrm{~min}$. Cool the mixture at room temperature. Added the 3-4 piece of ice or crushed ice having volume 3-4 times greater than the mixture with vigorous stirring, Yields was $72 \%$ melting point of different 5 -substituted Isatin. 5-ClIsatin- 254-256C $\mathrm{C}^{\circ}, \mathrm{NO}_{2}$ Isatin- 180-182 $\mathrm{C}^{\circ}, 5$-Fl-Isatin-224-226 $\mathrm{C}^{\circ}$, 5-I-Isatin-280-282 Co, 5- $\mathrm{CH}_{3}$-Isatin- 264-266 Co.

\section{R \& D of Schiff's bases of different 5-substituted 1-H-indole -2, 3-dione (Scheme II) (1a-p)}

Equimolar quantities $(0.004 \mathrm{~mol})$ of isatin/5-substituted isatin and the aromatic primary amine/hydrazine has dissolved in $10 \mathrm{~mL}$ of warm ethanol and heated on a steam bath for 20-40 min. standing for approximately $24 \mathrm{~h}$ at room temperature, the crystalline product has separated by filtration, vacuum dried and recrystallized from ethanol (Scheme 2).
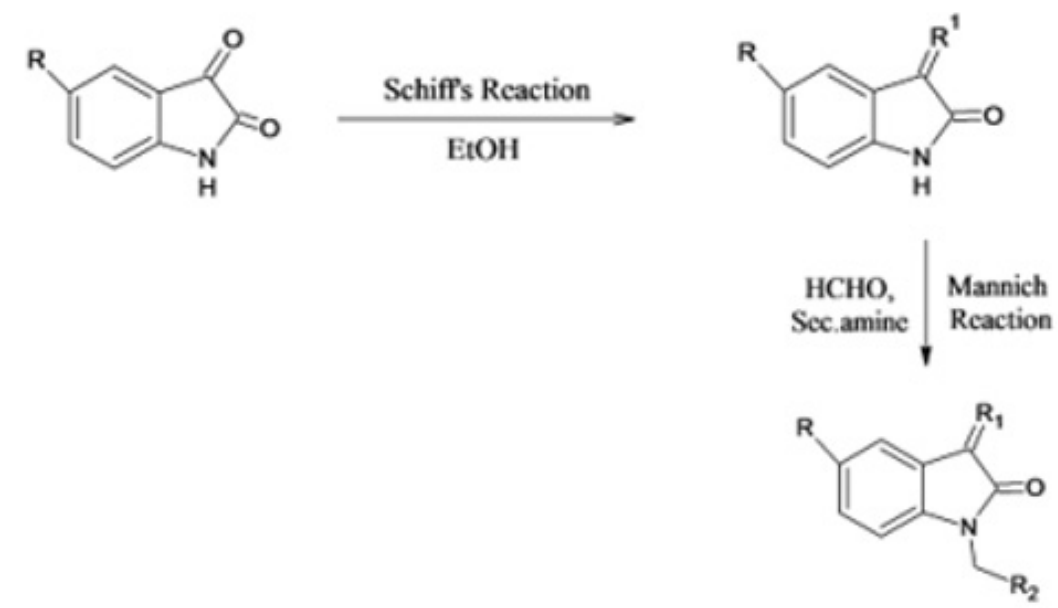

Scheme 2: Synthesis of Schiff' base of different 5-substituted 1-H-Indole -2, 3-dione followed by synthesis of different Mannich base from Schiff's base.

R \& D of different Mannich base from Schiff's base of different 1-H-Indol -2, 3-Dione (Scheme II) (1a-p)

Molar absolute $(0.004 \mathrm{~mol})$ of diphenyl amine in $10 \mathrm{~mL}$ of ethanol has added to slurry containing the appropriate isatin and aqueous formaldehyde solution dissolved in $10 \mathrm{~mL}$ of ethanol.
The reaction mixture has magnetically stirred for $1 \mathrm{~h}$ at room temperature and refrigerated for $48 \mathrm{~h}$. The product has separated by suction filtration, vacuum dried and recrystallized from ethanol. Final compound with their respective substitution of groups describe in Table 1 and physicochemical data of synthesized more novel 5-substituted 1-H-Indole-2,3-Dione described in Table 2.

Table 1: Showing compound code along with their respective substitution of groups.

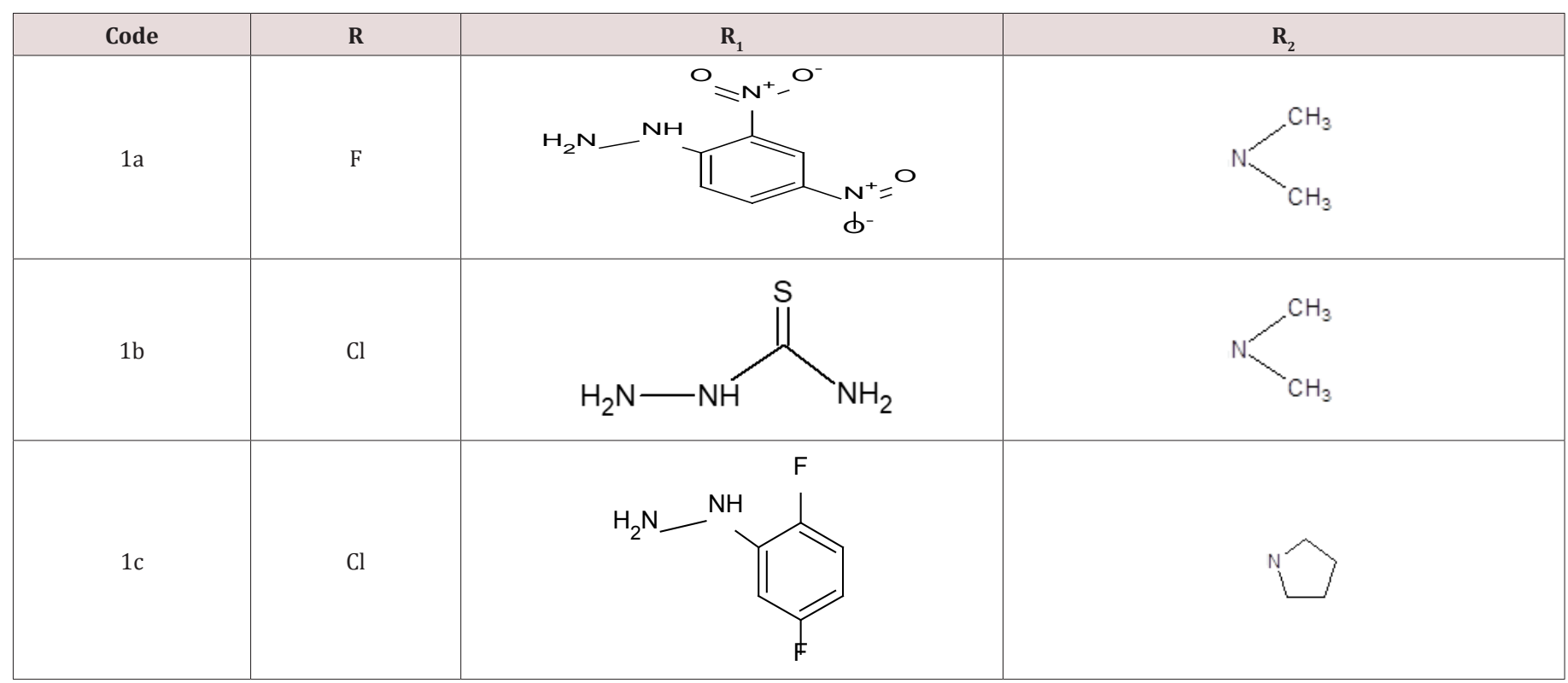




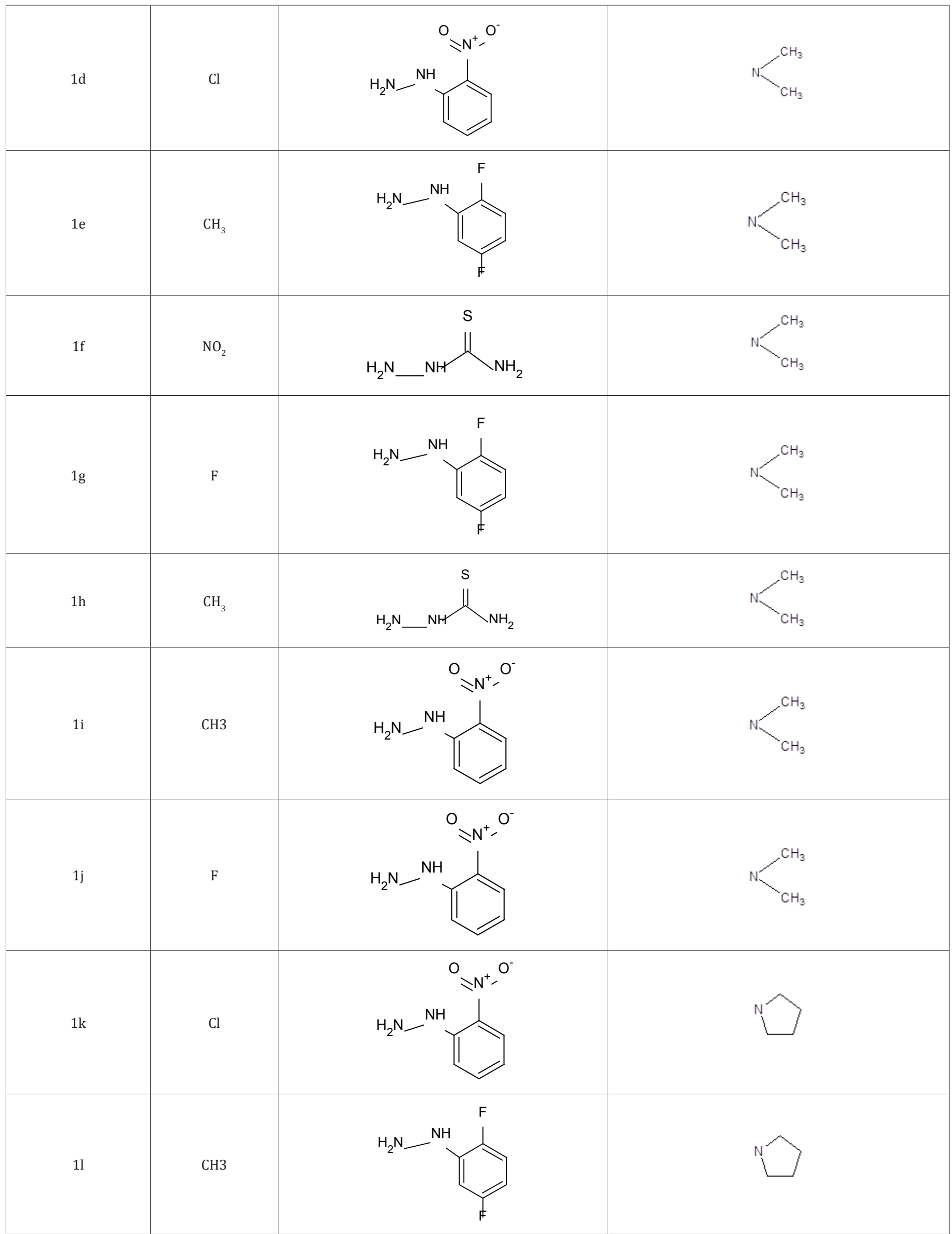




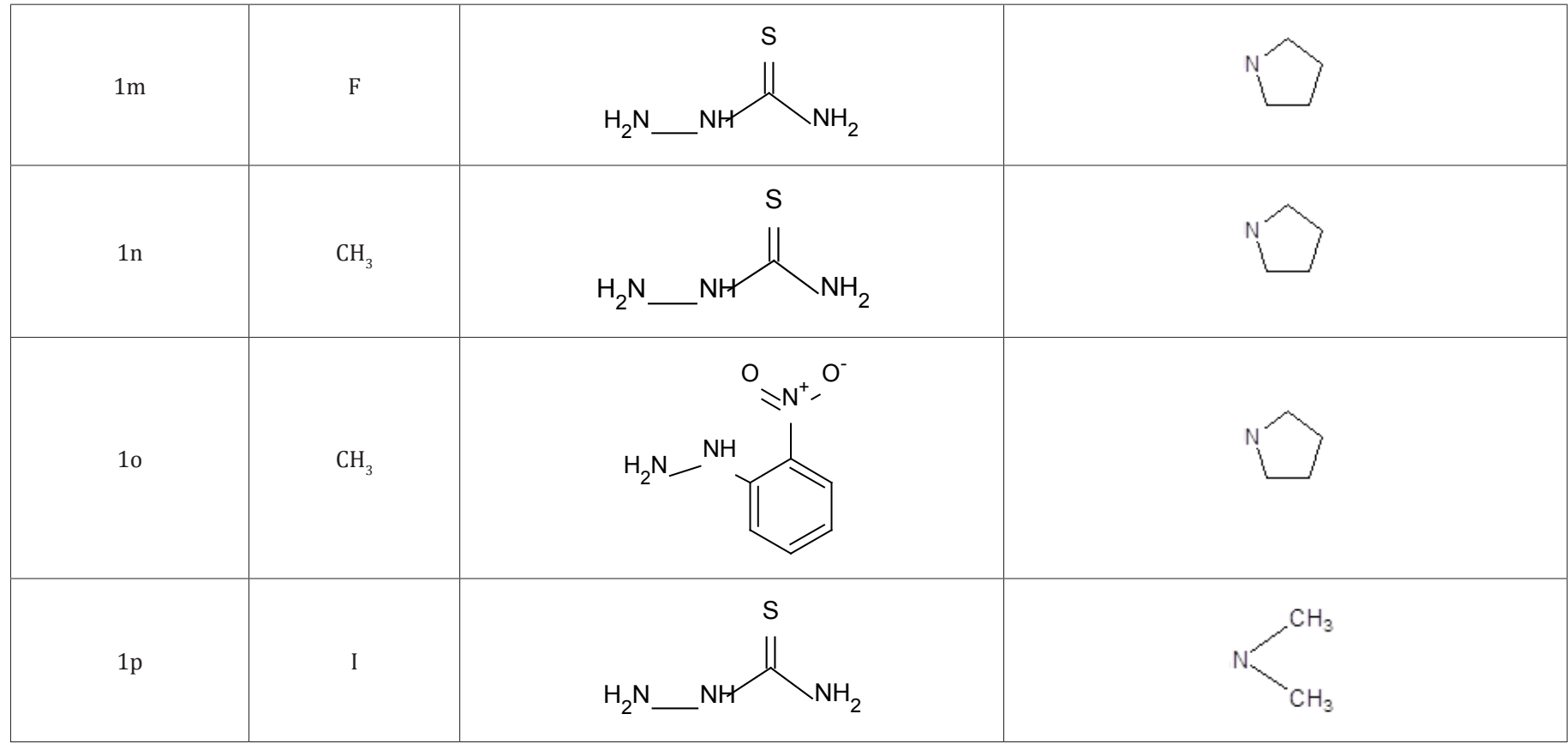

Table 2: Physicochemical Data of synthesized different 5-substituted 1-H-indole-2,3-dione.

\begin{tabular}{|c|c|c|c|c|c|}
\hline Code & Yield (\%) & M.P. $\left({ }^{0} \mathrm{C}\right)$ & Texture & M. F. & Rf value \\
\hline $1 \mathrm{a}$ & 62.5 & $378-380$ & Brown amorphous powder & $\mathrm{C}_{17} \mathrm{H}_{15} \mathrm{~N}_{6} \mathrm{O}_{5}$ & 0.47 \\
\hline $1 \mathrm{~b}$ & 63.7 & $274-276$ & Yellowish amorphous powder & $\mathrm{C}_{12} \mathrm{H}_{14} \mathrm{Cl} \mathrm{N}_{5} \mathrm{OS}$ & 0.24 \\
\hline $1 \mathrm{c}$ & 61.4 & $178-180$ & Yellowish amorphous powder & $\mathrm{C}_{19} \mathrm{H}_{17} \mathrm{Cl} \mathrm{F}_{2} \mathrm{~N}_{4} \mathrm{O}$ & 0.67 \\
\hline $1 \mathrm{~d}$ & 59.3 & $336-338$ & Dark brown powder & $\mathrm{C}_{17} \mathrm{H}_{16} \mathrm{Cl} \mathrm{N}_{5} \mathrm{O}_{3}$ & 0.70 \\
\hline $1 \mathrm{e}$ & 65.7 & $268-270$ & Yellow crystal & $\mathrm{C}_{18} \mathrm{H}_{18} \mathrm{~F}_{2} \mathrm{~N}_{4} \mathrm{O}$ & 0.64 \\
\hline 1f & 59.2 & $228-230$ & Yellowish brown powder & $\mathrm{C}_{12} \mathrm{H}_{14} \mathrm{~N}_{6} \mathrm{O}_{3} \mathrm{~S}$ & 0.30 \\
\hline $1 \mathrm{~g}$ & 57.3 & $278-280$ & Yellowish brown powder & $\mathrm{C}_{17} \mathrm{H}_{15} \mathrm{~F}_{3} \mathrm{~N}_{4} \mathrm{O}$ & 0.82 \\
\hline $1 \mathrm{~h}$ & 62.5 & $286-288$ & Orange fine powder & $\mathrm{C}_{13} \mathrm{H}_{17} \mathrm{~N}_{5} \mathrm{OS}$ & 0.46 \\
\hline $1 \mathrm{i}$ & 63.7 & $316-318$ & Brown amorphous powder & $\mathrm{C}_{18} \mathrm{H}_{19} \mathrm{~N}_{5} \mathrm{O}_{3}$ & 0.61 \\
\hline $1 \mathrm{j}$ & 61.4 & $310-312$ & Dark brown powder & $\mathrm{C}_{17} \mathrm{H}_{16} \mathrm{FN}_{5} \mathrm{O}_{3}$ & 0.38 \\
\hline $1 \mathrm{k}$ & 59.3 & $336-338$ & Brown powder & $\mathrm{C}_{19} \mathrm{H}_{18} \mathrm{ClN}_{5} \mathrm{O}_{3}$ & 0.29 \\
\hline 11 & 65.7 & $168-170$ & Yellow amorphous powder & $\mathrm{C}_{20} \mathrm{H}_{20} \mathrm{~F}_{2} \mathrm{~N}_{4} \mathrm{O}$ & 0.55 \\
\hline $1 \mathrm{~m}$ & 59.2 & $290-292$ & Brown powder & $\mathrm{C}_{14} \mathrm{H}_{6}{ }_{6} \mathrm{FN}_{5} \mathrm{OS}$ & 0.26 \\
\hline $1 \mathrm{n}$ & 72.1 & $266-268$ & Orange fine powder & $\mathrm{C}_{15} \mathrm{H}_{19} \mathrm{~N}_{5} \mathrm{OS}$ & 0.32 \\
\hline 10 & 64.2 & $250-252$ & Brown powder & $\mathrm{C}_{20} \mathrm{H}_{21} \mathrm{~N}_{5} \mathrm{O}_{3}$ & 0.42 \\
\hline $1 \mathrm{p}$ & 73.01 & $198-200$ & Yellow powder & $\mathrm{C}_{12} \mathrm{H}_{14} \mathrm{~N}_{5} \mathrm{IOS}$ & 0.54 \\
\hline
\end{tabular}

a) Solvent for crystallization: ethanol.

b) Melting point of the compounds at decomposition.

c) Solvent system: benzene/methanol (9:1).

\section{Preparation of Solutions}

The PTZ solution $(90 \mathrm{mg} / \mathrm{kg}$ ) has prepared in saline solution. Sodium valporate $(300 \mathrm{mg} / \mathrm{kg})$, has used as standard drug. Various test compounds have suspended in carboxy methyl cellulose $(0.5 \%)$, suspensions have prepared freshly prior to the dosing.

\section{Selection of Animals}

Albino female mice of Wister strain weighing around 25$35 \mathrm{gm}$ has collected and housed at the animal house in groups of six animals per cage at standard laboratory conditions at room temperature with $12: 12 \mathrm{~h}$ light and dark cycle. All the animals 
have fed on standard laboratory diet and free access of water. The scientific animal ethical committee approved the present animal protocol [2]. The selected animals have kept in the cages for a week prior to dosing for all the limitations of the laboratory conditions.

\section{Acute Toxicity Studies}

$\mathrm{LD}_{50}$ of test compounds have performed as per the O.E.C.D guideline 423. Test compounds have suspended in $0.5 \%$ CMC solution. The compounds have administered orally at a dose level of

\section{Evaluation of antiepileptic activity (PTZ model)}

Table 3: All the results are expressed as Mean + S.D and were subjected to student t-test using Graph-pad

\begin{tabular}{|c|c|c|c|}
\hline S No & Compound & Mean Latency time (Min.) & $\begin{array}{l}\text { Duration of convulsion } \\
\text { Mean } \pm \text { S.D.(Min.) }\end{array}$ \\
\hline 1 & PTZ & 2.40 & $10.24 \pm 1.18$ \\
\hline 2 & $1 \mathrm{~b}-50 \mathrm{mg}$ & 3.12 & $13.28 \pm 1.10$ \\
\hline 3 & $1 \mathrm{~b}-100 \mathrm{mg}$ & 3.22 & $7.57 \pm 0.56$ \\
\hline 4 & $1 \mathrm{~b}-150 \mathrm{mg}$ & 7.54 & $5.74 \pm 0.50$ \\
\hline 5 & $1 \mathrm{~b}-300 \mathrm{mg}$ & $8.42 *$ & $1.96 \pm 0.48$ \\
\hline 6 & $1 \mathrm{c}-50 \mathrm{mg}$ & 2.40 & $13.56 \pm 0.58$ \\
\hline 7 & $1 \mathrm{c}-100 \mathrm{mg}$ & 3.10 & $9.047 \pm 0.45$ \\
\hline 8 & $1 \mathrm{c}-150 \mathrm{mg}$ & 7.2 & $5.69 \pm 0.47$ \\
\hline 9 & $1 \mathrm{c}-300 \mathrm{mg}$ & 8.12 & $2.7 \pm 0.44$ \\
\hline 10 & $1 \mathrm{f}-50 \mathrm{mg}$ & 2.24 & $13.13 \pm 0.99$ \\
\hline 11 & $1 \mathrm{f}-100 \mathrm{mg}$ & 2.12 & $8.72 \pm 0.03$ \\
\hline 12 & $1 \mathrm{f}-150 \mathrm{mg}$ & 5.4 & $6.10 \pm 0.49$ \\
\hline 13 & $1 \mathrm{f}-300 \mathrm{mg}$ & 6.23 & $2.67 \pm 066$ \\
\hline 14 & $1 \mathrm{~h}-50 \mathrm{mg}$ & 2.20 & $13.257 \pm 1.045$ \\
\hline 15 & $1 \mathrm{~h}-100 \mathrm{mg}$ & 2.56 & $7.587 \pm 0.445$ \\
\hline 16 & $1 \mathrm{~h}-150 \mathrm{mg}$ & 7.56 & $5.72 \pm 0.503$ \\
\hline 17 & $1 \mathrm{~h}-300 \mathrm{mg}$ & 8.44 & $1.93 \pm 0.511$ \\
\hline 18 & $1 \mathrm{j}-50 \mathrm{mg}$ & 2.18 & $13.25 \pm 0.80$ \\
\hline 19 & $1 \mathrm{j}-100 \mathrm{mg}$ & 2.30 & $9.43 \pm 0.12$ \\
\hline 20 & $1 \mathrm{j}-150 \mathrm{mg}$ & 6.54 & $5.96 \pm 0.34$ \\
\hline 21 & $1 \mathrm{j}-300 \mathrm{mg}$ & 7.42 & $2.71 \pm 0.46$ \\
\hline 22 & $11-50 \mathrm{mg}$ & 2.52 & $12.59 \pm 1.50$ \\
\hline 23 & $11-100 \mathrm{mg}$ & 3.12 & $8.67 \pm 0.35$ \\
\hline 24 & $11-150 \mathrm{mg}$ & 8.42 & $5.2 \pm 0.95$ \\
\hline 25 & $11-300 \mathrm{mg}$ & $10.23^{* *}$ & $1.89 \pm 0.29$ \\
\hline 26 & $1 \mathrm{~m}-50 \mathrm{mg}$ & 2.23 & $12.04 \pm 0.46$ \\
\hline 27 & $1 \mathrm{~m}-100 \mathrm{mg}$ & 3.10 & $8.12 \pm 0.02$ \\
\hline 28 & $1 \mathrm{~m}-150 \mathrm{mg}$ & 7.42 & $6.28 \pm 0.16$ \\
\hline 29 & $1 \mathrm{~m}-300 \mathrm{mg}$ & 8.42 & $2.59 \pm 0.38$ \\
\hline 30 & $1 \mathrm{n}-50 \mathrm{mg}$ & 2.40 & $13.55 \pm 0.57$ \\
\hline 31 & $1 \mathrm{n}-100 \mathrm{mg}$ & 3.24 & $8.92 \pm 0.38$ \\
\hline 32 & 1n-150mg & 8.54 & $5.31 \pm 0.10$ \\
\hline 33 & $1 \mathrm{n}-300 \mathrm{mg}$ & $9.48^{*}$ & $2.18 \pm 0.13$ \\
\hline
\end{tabular}




\begin{tabular}{|c|c|c|c|}
\hline 34 & $10-50 \mathrm{mg}$ & 3.22 & $11.09 \pm 0.97$ \\
\hline 35 & $10-100 \mathrm{mg}$ & 3.36 & $8.31 \pm 0.11$ \\
\hline 36 & $10-150 \mathrm{mg}$ & 8.52 & $5.57 \pm 0.64$ \\
\hline 37 & $10-300 \mathrm{mg}$ & $9.40 * *$ & $1.61 \pm 0.44$ \\
\hline 39 & $1 \mathrm{p}-50 \mathrm{mg}$ & 3.12 & $10.29 \pm 0.98$ \\
\hline 40 & 1p-100mg & 3.20 & $7.93 \pm 0.47$ \\
\hline 41 & $1 \mathrm{p}-150 \mathrm{mg}$ & 8.34 & $5.57 \pm 0.47$ \\
\hline 42 & $1 \mathrm{p}-300 \mathrm{mg}$ & $9.52^{* *}$ & $1.44 \pm 0.50$ \\
\hline 43 & Phynetoin & 0.00 & $0.00^{* * *}$ \\
\hline 44 & Diazepam & 0.00 & $0.00^{* * *}$ \\
\hline 45 & Sod.Valporate & 0.00 & $0.00^{* * *}$ \\
\hline
\end{tabular}

prism 4 software. Values are considered as significant when $\mathrm{P}<0.05$.

${ }^{*} \mathrm{P}<0.05$ vs. pentylenetetrazole control (PTZ, $90 \mathrm{mg} / \mathrm{kg}$ i.p.).

${ }^{* *} \mathrm{P}<0.01$ vs. pentylenetetrazole control (PTZ, $90 \mathrm{mg} / \mathrm{kg}$ i.p.); students t-test

*** $\mathrm{P}<0.001$ vs. pentylenetetrazole control (PTZ, 90 mg/kg i.p.

The compounds synthesized in the present investigation has subjected to pharmacological screening, to ascertain their anticonvulsant activity. The anti-convulsant activity was evaluated by using PTZ induced convulsion mice models [4,5]. In this method convulsion produce by intraperitoneal administration of pentylinetetrazole (PTZ) solution prepared in saline $(90 \mathrm{mg} /$ $\mathrm{kg}, 10 \mathrm{~mL} / \mathrm{kg}$ ) was recorded over a period of $30 \mathrm{~min}$, after pentylinetetrazole injection. The mice were treated orally with test $(50,100,150,300 \mathrm{mg} / \mathrm{kg})$ suspended in $0.5 \%$ CMC or standard drug (sodium valporate $300 \mathrm{mg} / \mathrm{kg}$, diazepam $4 \mathrm{mg} / \mathrm{kg}$, phenytoin 30mg/ $\mathrm{kg}$ ), 30 min before i.p. injection of pentylinetetrazole. The time of convulsant recorded and permitted to express the protection of mice. The evaluations of anti-convulsant of the compounds has been listed in Table 3.

\section{Statistical Analysis}

All the results have expressed as Mean \pm S.D and has subjected to student t-test using Graph-pad prism 4 software. Values have considered as significant when $\mathrm{P}<0.05$.

\section{Results and Discussion}

Synthesis of 5-substituted-1H Indole-2, 3 Diones have done by extended Sandmeyer method from $\mathrm{Cl}, \mathrm{NO}_{2}, \mathrm{I}, \mathrm{CH}_{3}$ and $\mathrm{F}$ aniline. The synthesized compounds have purified in ethanol. All these spectral analysis data showed the authenticity of the structure of the synthesized compounds. The mass spectra of compounds 1a-p showed molecular ion peaks $\mathrm{M}+$ at $\mathrm{m} / \mathrm{z}$ corresponding to their respective molecular masses, which agrees with their respective molecular formulas recorded in the Table 4. Anticonvulsant activity of the compounds $1 \mathrm{~b}, 1 \mathrm{c}, 1 \mathrm{f}, 1 \mathrm{~h}, 1 \mathrm{j}, 1 \mathrm{l}, 1 \mathrm{~m}, 1 \mathrm{n}, 1 \mathrm{o}, 1 \mathrm{p}$ has examined using PTZ induced convulsion mice models. In this method convulsion produce by intraperitoneal administration of pentylinetetrazole (PTZ) solution prepared in saline $(90 \mathrm{mg} /$

$\mathrm{kg}, 10 \mathrm{~mL} / \mathrm{kg}$ ) has recorded over a period of $30 \mathrm{~min}$, after pentylinetetrazole injection. The mice have treated orally with test $(50,100,150,300 \mathrm{mg} / \mathrm{kg})$ suspended in $0.5 \%$ CMC or standard drug (sod. valporate $300 \mathrm{mg} / \mathrm{kg}$ ), 30 min before i.p. injection of pentylinetetrazole. The time of convulsant recorded and permitted to express the protection of mice. The evaluations of anti-convulsant of the compounds have listed in Table 5.

Table 4: Spectral characterization of synthesized different 5-substituted 1-H-indole-2,3- dione. 1HNMR Spectral characterization of synthesized different 5-substituted 1-H-indole-2,3- dione.

\begin{tabular}{|c|c|}
\hline Comp & $\operatorname{Mass}(\mathrm{m} / \mathrm{z})$ \\
\hline $1 \mathrm{a}$ & $402.33\left(\mathrm{M}^{+}\right)$ \\
\hline $1 b$ & $311.79\left(\mathrm{M}^{+}\right)$ \\
\hline $1 \mathrm{c}$ & $390.81\left(\mathrm{M}^{+}\right)$ \\
\hline $1 d$ & $373.79\left(\mathrm{M}^{+}\right)$ \\
\hline $1 \mathrm{e}$ & $344.35\left(\mathrm{M}^{+}\right)$ \\
\hline $1 \mathrm{f}$ & $322.34\left(\mathrm{M}^{+}\right)$ \\
\hline $1 \mathrm{~g}$ & $348.32\left(\mathrm{M}^{+}\right)$ \\
\hline $1 \mathrm{~h}$ & $291.37\left(\mathrm{M}^{+}\right)$ \\
\hline $1 \mathrm{i}$ & $353.37\left(\mathrm{M}^{+}\right)$ \\
\hline $1 \mathrm{j}$ & $357.33\left(\mathrm{M}^{+}\right)$ \\
\hline $1 \mathrm{k}$ & $399.83\left(\mathrm{M}^{+}\right)$ \\
\hline 11 & $370.39\left(\mathrm{M}^{+}\right)$ \\
\hline $1 \mathrm{~m}$ & $321.37\left(\mathrm{M}^{+}\right)$ \\
\hline $1 \mathrm{n}$ & $317.40\left(\mathrm{M}^{+}\right)$ \\
\hline 10 & $379.41\left(\mathrm{M}^{+}\right)$ \\
\hline $1 \mathrm{p}$ & $403.24\left(\mathrm{M}^{+}\right)$ \\
\hline
\end{tabular}


Table 5: Anti-convulsant data of compounds.

\begin{tabular}{|c|c|}
\hline Code & Chemical shift \& nature of Proton $(\delta \mathrm{ppm})$ \\
\hline $1 \mathrm{a}$ & 14.03 (d, 1H, NH.), 11.28 (s, 1H, NH) 7.88 (dm 3H, ar.), 7.59-7.47 (m, 4H, ar.), 7.611 (d, 1H, ar.), 7.25 (d, 1H, ar.), 6.86 (d, $1 \mathrm{H}$, ar.). \\
\hline $1 \mathrm{~b}$ & 14.03 (d, 1H, NH.), 11.28 (s, 1H, NH), 7.88-7.84 (m 2H, ar.), 7.59-7.42 (m, 4H, ar.), 7.25-7.23 (m, 1H, ar.), 6.87-6.84 (m, 1H, ar.). \\
\hline $1 \mathrm{c}$ & $14.06(\mathrm{~d}, 1 \mathrm{H}, \mathrm{NH}),$.10.37 (s, 1H, NH), 7.55-7.49 (m 2H, ar.), 7.43 (t, 2H, ar.), 7.05 (d, 1H, ar.), 6.73 (d, 1H, ar.), 2.24 (s, 3H, $\left.\mathrm{CH}_{3}\right)$ \\
\hline $1 \mathrm{~d}$ & $\begin{array}{c}14.03(\mathrm{~s}, 1 \mathrm{H}, \mathrm{NH} .), 7.97 \text { (d 2H, ar.), } 7.83(\mathrm{~s}, 1 \mathrm{H}, \mathrm{ar}), 7.54-7.46(\mathrm{~m}, 3 \mathrm{H}, \mathrm{ar} .), 7.28-7.18(\mathrm{~m}, 6 \mathrm{H}, \mathrm{ar} .), 7.07 \text { (t, 1H, ar.), 6.75, (d, 1H, ar.), } 4.92(\mathrm{~s}, 2 \mathrm{H}, \\
\left.\mathrm{CH}_{2}\right) .\end{array}$ \\
\hline $1 \mathrm{e}$ & $\begin{array}{c}14.00(\mathrm{~s}, 1 \mathrm{H}, \mathrm{NH} .), 7.99(\mathrm{~d} 2 \mathrm{H}, \mathrm{ar}), 7.86(\mathrm{~s}, 1 \mathrm{H}, \mathrm{ar}), 7.57(\mathrm{t}, 1 \mathrm{H}, \mathrm{ar}), 7.48(\mathrm{t}, 1 \mathrm{H}, \mathrm{ar}), 7.29-7.21(\mathrm{~m}, 5 \mathrm{H}, \mathrm{ar}), 7.11(\mathrm{t}, 1 \mathrm{H}, \mathrm{ar} .), 6.74(\mathrm{~d}, 1 \mathrm{H}, \mathrm{ar}) \\
4.91\left(\mathrm{~s}, 2 \mathrm{H}, \mathrm{CH}_{2}\right) .\end{array}$ \\
\hline 1f & $\begin{array}{c}14.04(\mathrm{~s}, 1 \mathrm{H}, \mathrm{NH} .), 7.97 \text { (d, 2H, ar.), } 7.82(\mathrm{~s}, 1 \mathrm{H}, \mathrm{ar}), 7.46 \text { (t, 2H, ar.), } 7.48(\mathrm{t}, 1 \mathrm{H}, \mathrm{ar} .), 7.25-7.04(\mathrm{~m}, 5 \mathrm{H}, \mathrm{ar} .), 6.76 \text { (d, } 1 \mathrm{H}, \mathrm{ar} .), 4.87\left(\mathrm{~s}, 2 \mathrm{H}, \mathrm{CH}_{2}\right), \\
2.25\left(\mathrm{~s}, 3 \mathrm{H}, \mathrm{CH}_{3}\right) .\end{array}$ \\
\hline $1 \mathrm{~g}$ & 13.84 (s, $1 \mathrm{H}, \mathrm{NH}.), 7.79$ (d, 2H, ar.), 7.72 (s, $1 \mathrm{H}, \mathrm{ar}.), 7.49,(\mathrm{t}, 2 \mathrm{H}, \mathrm{ar}),$.7.40 (t, $2 \mathrm{H}$, ar.), $7.21\left(\mathrm{dm}, 4 \mathrm{H}, \mathrm{ar}\right.$ ), 6.58 (d, $1 \mathrm{H}$, ar.), $4.86\left(\mathrm{~s}, 2 \mathrm{H}, \mathrm{CH}_{2}\right)$ \\
\hline $1 \mathrm{~h}$ & $\begin{array}{c}14.04(\mathrm{~s}, 1 \mathrm{H}, \mathrm{NH}), 7.97(\mathrm{~d}, 2 \mathrm{H}, \mathrm{ar}), 7.82(\mathrm{~s}, 1 \mathrm{H}, \mathrm{ar}), 7.54(\mathrm{t}, 2 \mathrm{H}, \mathrm{ar} .), 7.46(\mathrm{t}, 2 \mathrm{H}, \mathrm{ar} .), 7.25-7.04\left(\mathrm{~m}, 5 \mathrm{H}, \text { ar.), } 6.76(\mathrm{~d}, 1 \mathrm{H}, \mathrm{ar}), 4.87\left(\mathrm{~s}, 2 \mathrm{H}, \mathrm{CH}_{3}\right),\right. \\
2.25\left(\mathrm{~s}, 3 \mathrm{H}, \mathrm{CH}_{3}\right) .\end{array}$ \\
\hline $1 \mathrm{i}$ & $\begin{array}{c}14.08(\mathrm{~s}, 1 \mathrm{H}, \mathrm{NH}), 7.55-7.04(\mathrm{~d}, 2 \mathrm{H}, \mathrm{ar} .), 7.72(\mathrm{~s}, 1 \mathrm{H}, \mathrm{ar}), 7.60(\mathrm{t}, 2 \mathrm{H}, \mathrm{ar} .), 7.53(\mathrm{t}, 2 \mathrm{H}, \mathrm{ar} .), 7.37-7.29(\mathrm{~m}, 5 \mathrm{H}, \mathrm{ar}), 7.10(\mathrm{~d}, 1 \mathrm{H}, \text { ar.), } 6.99(\mathrm{~d}, 1 \mathrm{H}, \\
\text { ar.), } 4.96\left(\mathrm{~s}, 2 \mathrm{H}, \mathrm{CH}_{3}\right), 2.34\left(\mathrm{~s}, 3 \mathrm{H}, \mathrm{CH}_{3}\right) .\end{array}$ \\
\hline $1 \mathrm{j}$ & $\begin{array}{c}13.95(\mathrm{~s}, 1 \mathrm{H}, \mathrm{NH}), 7.96(\mathrm{~d}, 2 \mathrm{H}, \mathrm{ar} .), 7.66(\mathrm{~s}, 1 \mathrm{H}, \mathrm{ar}), 7.54(\mathrm{t}, 1 \mathrm{H}, \mathrm{ar}), 7.46(\mathrm{t}, 1 \mathrm{H}, \mathrm{ar} .), 7.25-7.17(\mathrm{~m}, 4 \mathrm{H}, \mathrm{ar} .), 7.05 \text { (d, } 1 \mathrm{H}, \text { ar.), } 6.59(\mathrm{~d}, 1 \mathrm{H}, \mathrm{ar}) \\
4.85\left(\mathrm{~s}, 2 \mathrm{H}, \mathrm{CH}_{3}\right), 2.27\left(\mathrm{~s}, 3 \mathrm{H}, \mathrm{CH}_{3}\right) .\end{array}$ \\
\hline $1 \mathrm{k}$ & $\begin{array}{c}14.02(\mathrm{~s}, 1 \mathrm{H}, \mathrm{NH}), 7.97(\mathrm{~d}, 2 \mathrm{H}, \mathrm{ar}), 7.64(\mathrm{~s}, 1 \mathrm{H}, \mathrm{ar}), 7.53(\mathrm{t}, 1 \mathrm{H}, \mathrm{ar} .), 7.45(\mathrm{t}, 2 \mathrm{H}, \mathrm{ar}), 7.25-7.01(\mathrm{~m}, 5 \mathrm{H}, \mathrm{ar}), 6.63(\mathrm{~d}, 1 \mathrm{H}, \mathrm{ar}), 4.85\left(\mathrm{~s}, 2 \mathrm{H}, \mathrm{CH}_{2}\right), \\
2.26\left(\mathrm{~s}, 6 \mathrm{H}, 2 \mathrm{CH}_{3}\right) .\end{array}$ \\
\hline 11 & 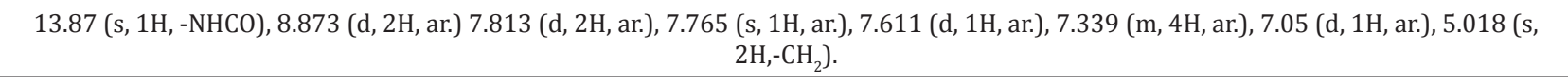 \\
\hline $1 \mathrm{~m}$ & $15.84(\mathrm{~s}, 1 \mathrm{H}, \mathrm{NH}),$.8.79 (d, 2H, ar.), $9.72(\mathrm{~s}, 1 \mathrm{H}, \mathrm{ar}), 8.49,.(\mathrm{t}, 2 \mathrm{H}, \mathrm{ar}),$.7.40 (t, $2 \mathrm{H}, \mathrm{ar}.), 7.21(\mathrm{dm}, 4 \mathrm{H}, \mathrm{ar}),. 6.58(\mathrm{~d}, 1 \mathrm{H}, \mathrm{ar}),. 4.86\left(\mathrm{~s}, 2 \mathrm{H}, \mathrm{CH}_{2}\right)$ \\
\hline $1 \mathrm{n}$ & $13.84(\mathrm{~s}, 1 \mathrm{H}, \mathrm{NH}),$.7.79 (d, $2 \mathrm{H}, \mathrm{ar}$ ), 7.72 (s, $1 \mathrm{H}, \mathrm{ar}.), 7.49,(\mathrm{t}, 2 \mathrm{H}, \mathrm{ar}),$.7.40 (t, $2 \mathrm{H}, \mathrm{ar}.), 7.21(\mathrm{dm}, 4 \mathrm{H}, \mathrm{ar}),$.6.58 (d, $1 \mathrm{H}, \mathrm{ar}.), 4.86\left(\mathrm{~s}, 2 \mathrm{H}, \mathrm{CH}_{2}\right)$ \\
\hline 10 & $\begin{array}{c}14.57(\mathrm{~s}, 1 \mathrm{H},-\mathrm{NHCO}), 7.873(\mathrm{~d}, 2 \mathrm{H}, \mathrm{ar}) 6.883(\mathrm{~d}, 2 \mathrm{H}, \mathrm{ar}), 8.965(\mathrm{~s}, 1 \mathrm{H}, \mathrm{ar}), 6.711(\mathrm{~d}, 1 \mathrm{H}, \mathrm{ar} .), 8.639(\mathrm{~m}, 4 \mathrm{H}, \mathrm{ar} .), 7.05(\mathrm{~d}, 1 \mathrm{H}, \operatorname{ar} .), 5.018(\mathrm{~s}, \\
\left.2 \mathrm{H},-\mathrm{CH}_{2}\right) .\end{array}$ \\
\hline $1 \mathrm{p}$ & 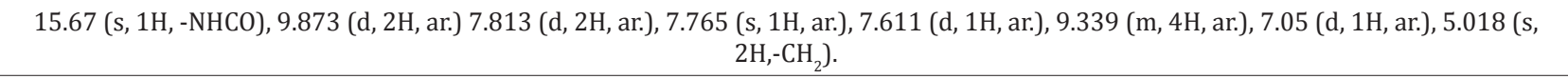 \\
\hline
\end{tabular}

\section{Conclusion}

It can be concluded that the Schiff and N-Mannich base of 5-substituted-1H Indole-2, 3 Diones has synthesized easily. The pharmacological screening has done by pentylenetetrazole (PTZ) induced convulsion method, for this Sodium. Valporate $(300 \mathrm{mg} /$ $\mathrm{kg}$ ) has used as reference standard. Test compound has given to mice in different doses $(50,100,150,300 \mathrm{mg} / \mathrm{kg})$. The result suggested that all the compounds possess good anti-convulsant activity with increased in latency time and reduction in duration of convulsion as like the standard drug when compared Table 3. All tested compound shows moderate effect at $150 \mathrm{mg} / \mathrm{kg}$ dose and significantly effective at $300 \mathrm{mg} / \mathrm{kg}$ dose. It concluded that the synthesized Schiff and N-Mannich isatin derivatives have shown excellent anti-convulsant activity.

\section{References}

1. Verma M, Pandeya S, Singh k, StableS J (2004) Anticonvulsant activity of Schiff bases of isatin derivatives, Acta Pharm 54(1): 49-56.

2. Lowenstein H (2001) Seizures and Epilepsy in Harrison's principles of Internal medicine $15^{\text {th }}$ Edn 2: 2354-2368.

3. Losher W (1993) Sdimdt D: Central \& Peripheral nervous system: New drugs for treatment of epilepsy curr Opin Invest Drugs 2: 1067-1095.

4. Pajouhesh H, Parson R, POPP FD 72 (1983) Potential anticonvulsants VI: Condensation of isatin with cyclohexanone and other cyclic ketones, J Pharm Sci 72(3): 318-321.

5. N Karali, Singh K (1994) Synthesis and anticonvulsant activity of some new thio semi carbazone and 4-thiazolidine derivatives bearing an isatin moiety, Farmaco 49(12): 819-822. 
(C) (1) This work is licensed under Creative

To Submit Your Article Click Here: Submit Article

DOI: $10.32474 /$ AOICS.2018.03.000165

\section{AOICS}

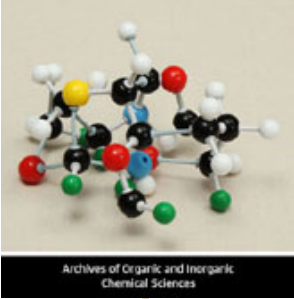

\section{Archives of Organic and Inorganic} Chemical Sciences

Assets of Publishing with us

- Global archiving of articles

- Immediate, unrestricted online access

- Rigorous Peer Review Process

- Authors Retain Copyrights

- Unique DOI for all articles 